\title{
Calibration of Collaborative Robots Based on Position Information and Local Product of Exponentials
}

\author{
Guanbin Gao $\mathbb{D}$, Yangtao Gao $\mathbb{D}$, Fei Liu $\mathbb{D}$, and Jing Na $\mathbb{D}$ \\ Faculty of Mechanical and Electrical Engineering, Kunming University of Science and Technology, Kunming 650500, China
}

Correspondence should be addressed to Fei Liu; feiliu2017@foxmail.com

Received 29 October 2021; Revised 17 December 2021; Accepted 28 January 2022; Published 4 March 2022

Academic Editor: Xue-bo Jin

Copyright (C) 2022 Guanbin Gao et al. This is an open access article distributed under the Creative Commons Attribution License, which permits unrestricted use, distribution, and reproduction in any medium, provided the original work is properly cited.

\begin{abstract}
To improve the positioning accuracy of collaborative robots, a novel modeling and calibration method for collaborative robots is proposed based on position information and modified local product of exponentials (LPoE). The kinematic error model is derived from the kinematic model through differential transformation. To solve the problem of the high redundancy and complexity of the error model that is difficult to identify, the errors of the kinematic parameters are all transferred to the initial position and posture matrix of the local coordinate system, which simplifies the error model and improves the speed and accuracy of the identification calculation. However, the simplified error model still requires posture data which are very difficult to acquire in practice. For this reason, the position error is separated from the kinematic model, and an error model based on position data is established. Then, a kinematic calibration method of the collaborative robot based on position data is proposed, which simplifies the measurement process and improves the efficiency of calibration. The effectiveness of the method is verified by simulations and experiments on a six degree-of-freedom collaborative robot.
\end{abstract}

\section{Introduction}

With the development of robots and the change of manufacturing mode, the working field of robots is expanding to realize natural interaction with humans, e.g., collaborative robots begin to be applied in unstructured environments to cooperate with humans. The collaborative robot is a new type of industrial robots, which can directly interact with people in the designated cooperative area [1]. Compared with traditional industrial robots, collaborative robots have the advantages of high security, universality, sensitivity, easy to use, and man-machine cooperation, which can be applied in the field of industrial production, family services, and rehabilitation medicine [2-4]. Generally, the actual kinematic parameters of the robot are inconsistent with the nominal parameters in its controller for the error of manufacturing and assembly and the stress deformation of joints and linkages, which results in control errors and reduces the positioning accuracy of the robot. Moreover, the collaborative robot is an open-chain mechanism with low stiffness and error accumulation and amplification. Therefore, the accuracy of the collaborative robot is much lower than that of traditional orthogonal instruments, and it cannot be applied to cases with high accuracy requirements, such as measuring, precision assembly, turning, and milling $[5,6]$.

Kinematic calibration is one of the main methods to improve the absolute positioning accuracy of robots, which is the process of identifying the actual kinematic parameters of the robots by using advanced measurement means and model-based parameter identification method, and compensating them to the controller of robots or compensating to improve positioning accuracy [7]. Kinematic calibration has been a research hotspot in the field of robots [8-10], which consists of four steps:kinematic modeling, pose measuring, parameter identification, and compensation [11]. Most kinematic models in the controller of robots are based on Denavit-Hartenberg (D-H) method [12]. However, when two adjacent axes of the robot are parallel, the Jacobian matrix of the D-H model will be singular [13]; that is, the kinematic solution has abrupt changes and discontinuities, which results in coupling problem between some of the kinematic parameters [14]. To solve the singularity, Park et al. [15] proposed a kinematic modeling method, i.e., 
PoE, which only needs to build the base and tool coordinate systems on the $0^{\text {th }}$ linkage and the end. Nevertheless, the PoE error model contains a finite integral term, which is not an explicit form and is inconvenient to use leading to the identifiability of its kinematic parameters cannot be determined [16]. He et al. [17] modified the PoE model by deriving the differential solution formula of exponential mapping on joint twists and variables and established an error model based on PoE model with explicit expression, which is the main PoE method used in kinematic calibration at present. Jiang et al. [18] used the PoE model to analyze the position space relationship of joints' axis under different geometric errors and proposed a calibration model including joint errors. Wang et al. [19] proposed a self-kinematic system for an on-orbit space manipulator based on PoE formula, which can measure the position and posture of the manipulator without using external measurement devices.

In the kinematic parameter identification of robots, the $\mathrm{D}-\mathrm{H}$ model only needs the end position data of robots, while the traditional PoE model also needs to obtain the position and posture data of robots. It is difficult to obtain posture data in practice. The operation is cumbersome, time-consuming, and difficult to ensure its accuracy [20]. Therefore, the traditional PoE model is difficult to be applied in the actual kinematic calibration of robots.

There are $13 \times n$ parameters in the traditional PoE-based error model which is very difficult to be identified. In this paper, to simplify the error model and improve the redundancy, the error model is simplified by transferring all the errors of the kinematic parameters to the initial position and posture matrix, to avoid the use of posture data which is difficult to obtain. The position error is separated from the kinematic model, and an error model based on position data is established. Then, a kinematic calibration method of collaborative robot based on position data is proposed, which simplifies the measurement process and improves the efficiency of calibration.

\section{Kinematic Modeling based on LPoE for the Collaborative Robot}

In this section, the transformation of the local coordinate system of the adjacent linkages is derived, based on which the LPoE kinematic model of a typical six degree-offreedom collaborative robot is established.

2.1. Description of the Coordinate System of Adjacent Linkages. Figure 1 shows a typical six degree-of-freedom collaborative robot. Unlike general industrial robots, the axes of the second, third, and fourth joints of the collaborative robot are parallel to each other.

Like the D-H model, the LPoE model uses a local coordinate system to describe the motion relationship of adjacent links [21]. As shown in Figure 2, the relationship between the adjacent linkages $i-1$ and $i$ can be written as (1).

$$
T_{i-1, i}\left(q_{i}\right)=T_{i-1, i}(0) e^{\widehat{s}_{i} q_{i}},
$$

where $T_{i-1, i}(0) \in S E(3)$ is the initial transformation matrix

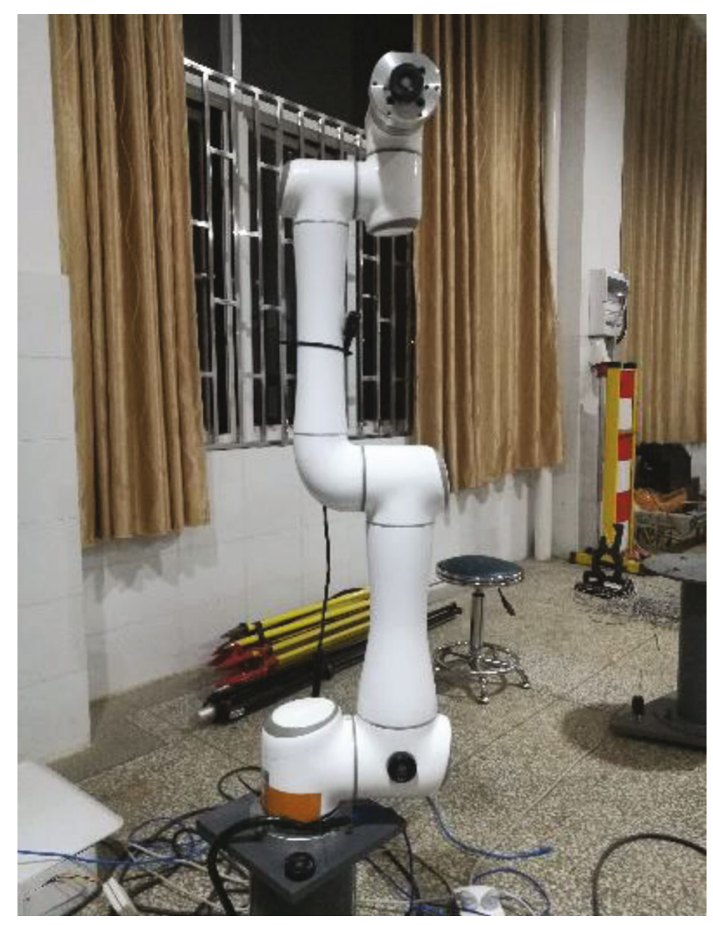

Figure 1: The six degree-of-freedom collaborative robot.

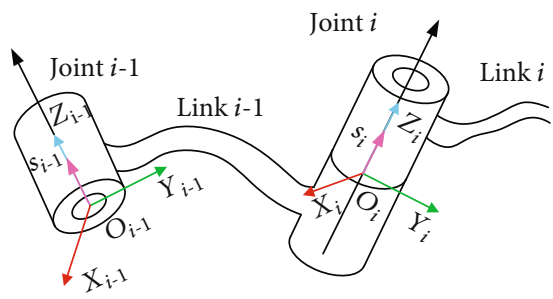

Figure 2: The coordinate systems of adjacent joints.

between coordinate systems of $i$ and $i-1, \widehat{s}_{i} \in s o(3)$ denotes the twist of joint $i, q_{i}$ denotes the rotation angle of joint $i$, and $e^{\widehat{s}_{i}} q_{i}$ is a matrix:

$$
e^{\hat{s}_{i} q_{i}}=\left[\begin{array}{cc}
e^{\hat{w}_{i} q_{i}} & v_{i} q_{i} \\
0 & 1
\end{array}\right],
$$

where $e^{\widehat{w}_{i} q_{i}}=I+\widehat{w}_{i} \sin q_{i}+\widehat{w}_{i}^{2}\left(1-\cos q_{i}\right)$.

Furthermore, $\widehat{s}_{i}$ can be written in the coordinate system $i$

$$
\widehat{s}_{i}=\left[\begin{array}{cc}
\widehat{w}_{i} & v_{i} \\
0 & 0
\end{array}\right],
$$

where $v_{i}=\left[v_{i x}, v_{i y}, v_{i z}\right]^{\mathrm{T}} \in \mathbb{R}^{3 \times 1}$ and $\widehat{w}_{i} \in s o(3)$ are the crossproduct matrixes of $w_{i}=\left[w_{i x}, w_{i y}, w_{i z}\right] \in \mathbb{R}^{3 \times 1} . \widehat{w}_{i}$ can be 
written as

$$
\widehat{w}_{i}=\left[\begin{array}{ccc}
0 & -w_{i z} & w_{i y} \\
w_{i z} & 0 & -w_{i x} \\
-w_{i y} & w_{i x} & 0
\end{array}\right] .
$$

To be consistent with existing robot position and posture description methods, $T_{i-1, i}(0) \in S E(3)$ can be written as the form of position and posture matrix:

$$
T_{i-1, i}(0)=\left[\begin{array}{cc}
R_{i-1, i}(0) & d_{i-1, i}(0) \\
0 & 1
\end{array}\right], \quad 7
$$

where $R_{i-1, i}(0) \in S O(3)$ and $d_{i-1, i}(0) \in \mathbb{R}^{3 \times 1}$ denote the initial posture and position, respectively.

2.2. Kinematic Modeling for the Robot. Based on the coordinate systems of adjacent joints, the kinematic model of the six degree-of-freedom collaborative robot can be derived as follows:

$$
\begin{aligned}
T_{0,6}\left(q_{1}, q_{2}, \cdots, q_{6}\right) & =T_{0,1}\left(q_{1}\right) T_{1,2}\left(q_{2}\right) \cdots T_{5,6}\left(q_{6}\right) \\
& =T_{0,1}(0) e^{\widehat{s}_{1} q_{1}} T_{1,2}(0) e^{\hat{s}_{2} q_{2}} \cdots T_{5,6}(0) e^{\widehat{s}_{6} q_{6}}
\end{aligned}
$$

$T_{6,7}$ is used to denote the transformation from the flange to the tool of the robot. Then, the position and posture matrix of the tool will be

$$
T_{0,7}\left(q_{1}, q_{2}, \cdots, q_{6}\right)=T_{0,1}(0) e^{\widehat{s}_{1} q_{1}} T_{1,2}(0) e^{\widehat{s}_{2} q_{2}} \cdots T_{5,6}(0) e^{\hat{s}_{6}} q_{6} T_{6,7} .
$$

Following the method of LPoE, the coordinate systems of the robot were established as shown in Figure 3. The nominal value of the twists is $s_{1}=s_{2}=s_{3}=s_{4}=s_{5}=s_{6}=$ $\left[\begin{array}{llllll}0 & 0 & 1 & 0 & 0 & 0\end{array}\right]^{T}$. The nominal kinematic parameters can be determined according to the structural size of the robot, as shown in Table 1.

\section{The Error Modeling Based on LPoE for the Collaborative Robot}

In this section, the error modeling of the collaborative robot based on LPoE is proposed and simplified. Then, the posebased and position-based error models are derived, respectively. The identification method and flow chart based on the least square method are presented.

3.1. Error Modeling and Simplifying. The kinematic model, i. e., (7) can be written as the function of $T(0), s$, and $q$ :

$$
T=f(T(0), s, q)
$$

where $\quad T(0)=\left[T_{0,1}(0), T_{1,2}(0), \cdots, T_{6,7}(0)\right]^{T}, \quad s=$ $\left[s_{1}, s_{2}, \cdots, s_{6}\right]^{T}$, and $q=\left[q_{1}, q_{2}, \cdots, q_{6}\right]^{T}$.

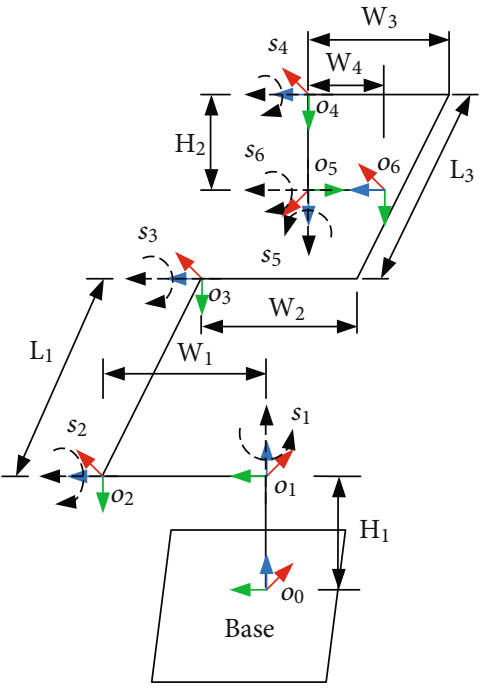

FIGURE 3: The coordinate systems and twists of the robot.

TABLE 1: The kinematic parameters of the robot.

\begin{tabular}{lcccccccc}
\hline Name & $H_{1}$ & $W_{1}$ & $\mathrm{~L}_{1}$ & $W_{2}$ & $\mathrm{~L}_{2}$ & $W_{3}$ & $H_{2}$ & $W_{4}$ \\
\hline Value $[\mathrm{mm}]$ & 96 & 138 & 418 & 114 & 398 & 98 & 98 & 89 \\
\hline
\end{tabular}

By linearization of (8), the error model can be derived:

$$
\delta T T^{-1}=\left(\frac{\partial f}{\partial T(0)} \delta T(0)+\frac{\partial f}{\partial s} \delta s+\frac{\partial f}{\partial q} \delta q\right) T^{-1},
$$

where $\delta T T^{-1}$ denotes the error of position and posture of the tool in the base coordinate system, which can be calculated by subtracting the measuring position and posture from the nominal ones. Kinematic parameters can be obtained by identification with the measuring data and the error model. The objective function of the identification model is generally set as the least square solution, i.e.,

$\operatorname{Min}\left(\sum\left\|\delta T T^{-1}-\left(\frac{\partial f}{\partial T(0)} \delta T(0)+\frac{\partial f}{\partial s} \delta s+\frac{\partial f}{\partial q} \delta q\right) T^{-1}\right\|\right)$.

There are 13 parameters to be identified in each joint in (10), and there are 6 joints in the collaborative robot. So, many parameters make the parameter identification model highly redundant and complicated. It is very difficult to directly identify the parameters.

To solve the problem of high redundancy and complexity of the error model based on LPoE, the simplified error model will be presented. As shown in Figure 4, the nominal coordinate system of joint $i\left\{o_{i} x_{i} y_{i} z_{i}\right\}$ and the actual coordinate system $\left\{o_{i}{ }^{a} x_{i}{ }^{a} y_{i}{ }^{a} z_{i}{ }^{a}\right\}$ are not coincident for the error of kinematic parameters, i.e., the nominals $T_{i-1, i}(0), s_{i}$ and $q_{i}$ are not equal to the actual $T^{a}{ }_{i-1, i}(0), s_{i}{ }^{a}$, and $q_{i}{ }^{a}$. For the local coordinate system of LPoE can be assigned arbitrarily, the following two assumptions are proposed [21]: (1) the kinematic error only exists in the initial position and posture 


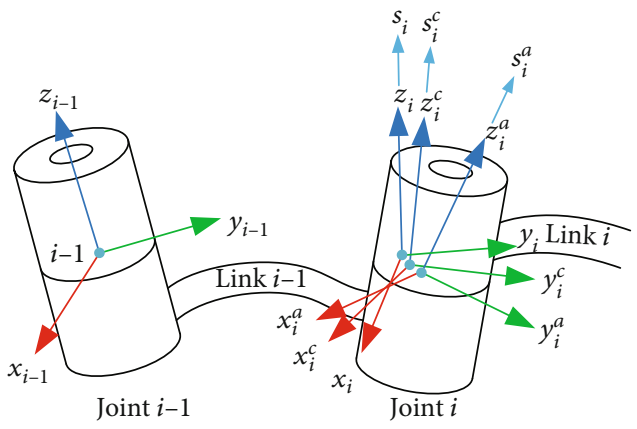

FIGURE 4: The error of kinematic parameters in coordinate systems.

of the local coordinate system $T(0)$, and (2) the nominal values of $q_{i}$ and $s_{i}$ remain unchanged in the whole parameter identification calculation process.

Based on these assumptions, a new local coordinate system $T_{i}^{c}(0)=\left\{o_{i}{ }^{c} x_{i}^{c} y_{i}^{c} z_{i}^{c}\right\}$ is set at each joint as the intermedia coordinate system in the following identification, as shown in Figure 4. Then, $q_{i}{ }^{c}$ and $s_{i}{ }^{c}$ will remain unchanged as $q_{i}$ and $s_{i}$ in the identification. Therefore, (9) and (10) can be simplified as

$$
\begin{gathered}
\delta T T^{-1}=\frac{\partial f}{\partial T(0)} \delta T(0) T^{-1}, \\
\operatorname{Min}\left(\sum\left\|\delta T T^{-1}-\frac{\partial f}{\partial T(0)} \delta T(0) T^{-1}\right\|\right) .
\end{gathered}
$$

3.2. Error Modeling Based on Position and Posture. According to Chasles theorem [22], for a given homogeneous transformation matrix $T \in S E(3)$, there is at least one coordinate twist $\widehat{p} \in \operatorname{se}(3)$ such that $e^{\widehat{p}}=T$. Then, one can obtain (12) according to (1).

$$
e^{\widehat{p}_{i}}=T_{i-1, i}(0)
$$

where $\widehat{p}_{i} \in \operatorname{se}(3)(i=1,2, \cdots, 7)$. Then, (7) can be rewritten as

$$
T_{0,7}=e^{\widehat{p}_{1}} e^{\widehat{S}_{1} q_{1}} e^{\widehat{p}_{2}} e^{\widehat{s}_{2} q_{2}} \cdots e^{\hat{p}_{6}} e^{\hat{s}_{6} q_{6}} e^{\hat{p}_{7}}
$$

Let $\delta \widehat{p}_{i}$ denote the error of $\widehat{p}_{i}$. Then, one can obtain $\delta \widehat{p}_{i}=$ $e^{-\widehat{p}_{i}} \delta\left(e^{\hat{p}_{i}}\right)$ and $\delta\left(e^{\hat{p}_{i}}\right)=e^{\hat{p}_{i}} \delta \widehat{p}_{i}$. By linearization of (13), one can get

$$
\begin{aligned}
& \delta T_{0,7}=\delta\left(e^{\widehat{p}_{1}}\right) e^{\hat{s}_{1} q_{1}} e^{\hat{p}_{2}} e^{\hat{s}_{2} q_{2}} \cdots e^{\hat{p}_{6}} e^{\hat{s}_{6}} q_{6} e^{\hat{p}_{7}} \\
& +e^{\hat{p}_{1}} e^{\hat{s}_{1} q_{1}} \delta\left(e^{\hat{p}_{2}}\right) e^{\hat{s}_{2} q_{2}} \cdots e^{\hat{p}_{6}} e^{\hat{s}_{6} q_{6}} e^{\hat{p}_{7}}+\cdots \\
& +e^{\widehat{p}_{1}} e^{\hat{s}_{1} q_{1}} \cdots e^{\widehat{p}_{5}} e^{\hat{s}_{5} q_{5}} \delta\left(e^{\hat{p}_{6}}\right) e^{\hat{s}_{6} q_{6}} e^{\hat{p}_{7}} \\
& +e^{\hat{p}_{1}} e^{\hat{s}_{1} q_{1}} \cdots e^{\hat{p}_{6}} e^{\hat{s}_{6} q_{6}} \delta\left(e^{\hat{p}_{7}}\right) \\
& =e^{\hat{p}_{1}} \delta \widehat{p}_{1} e^{\hat{s}_{1} q_{1}} e^{\hat{p}_{2}} e^{\hat{s}_{2} q_{2}} \ldots e^{\hat{p}_{6}} e^{\hat{s}_{6} q_{6}} e^{\hat{p}_{7}} \\
& +e^{\widehat{p}_{1}} e^{\hat{s}_{1} q_{1}} e^{\hat{p}_{2}} \delta \widehat{p}_{2} e^{\hat{s}_{2} q_{2}} \cdots e^{\hat{p}_{6}} e^{\hat{s}_{6} q_{6}} e^{\hat{p}_{7}} \\
& +\cdots+e^{\widehat{p}_{1}} e^{\hat{s}_{1} q_{1}} \cdots e^{\hat{p}_{5}} e^{\hat{s}_{5} q_{5}} e^{\hat{p}_{6}} \delta \widehat{p}_{6} e^{\hat{s}_{6} q_{6}} e^{\hat{p}_{7}} \\
& +e^{\widehat{p}_{1}} e^{\widehat{s}_{1} q_{1}} \cdots e^{\widehat{p}_{6}} e^{\widehat{s}_{6} q_{6}} e^{\widehat{p}_{7}} \delta \widehat{p}_{7} .
\end{aligned}
$$

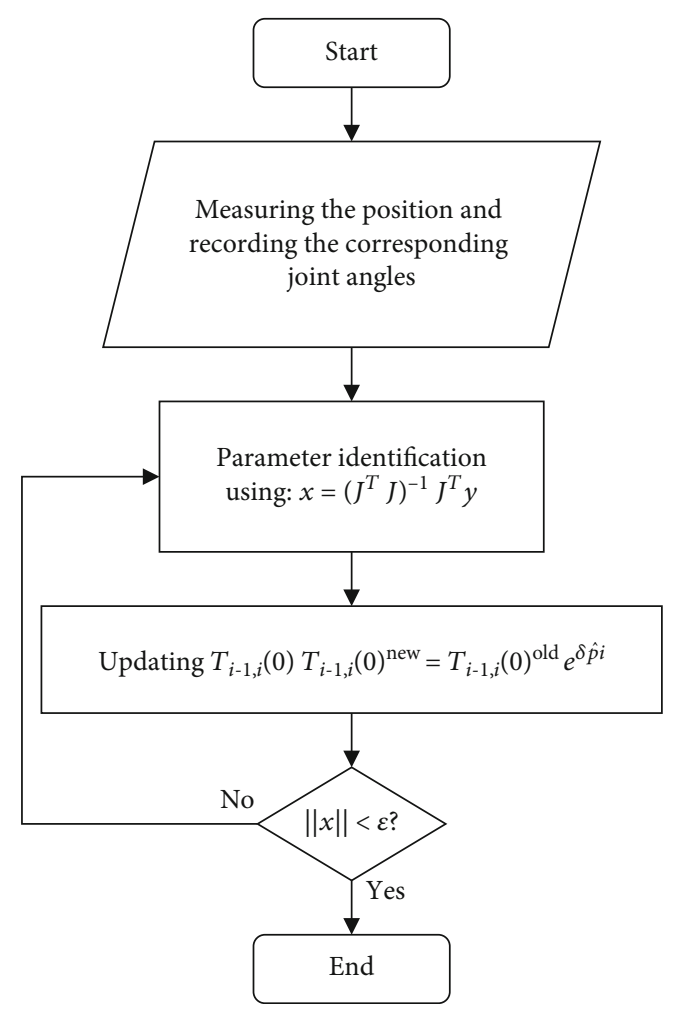

FIgURE 5: The flow chart of kinematic parameters identification by the iterative least square method.

Right multiplying $T_{0,7}^{-1}$ both sides of (14), one can get (15) in the form of adjoint transformation.

$$
\begin{aligned}
& \delta T_{0,7} T_{0,7}^{-1}=\operatorname{Ad}_{\hat{e}_{1}^{\hat{p}_{1}}} \delta \widehat{p}_{1}+\operatorname{Ad}_{\hat{e}_{1}^{\hat{p}_{1}} \hat{\hat{\vartheta}}_{1} q_{1}} \operatorname{Ad}_{e_{\hat{e}_{2}} \delta} \delta \widehat{p}_{2}+\cdots
\end{aligned}
$$

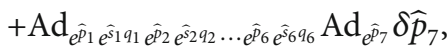

where $\delta T_{0,7} T_{0,7}^{-1} \in S E(3)$ denotes the total error of kinematic parameters of the robot relative to the base coordinate system. Ad is the adjoint transformation defined as

$$
\operatorname{Ad}: S E(3) \mapsto \Re^{6 \times 6}, g=\left[\begin{array}{cc}
R & P \\
0 & 1
\end{array}\right] \mapsto \operatorname{Ad}_{g}=\left[\begin{array}{cc}
R & \widehat{P} R \\
0 & R
\end{array}\right],
$$

where $g$ is a homogeneous transformation matrix, $R$ is the orthogonal matrix in $g$, and $\widehat{P}$ is the antisymmetric matrix of $P$. According to the matrix logarithm calculation formula defined in SE (3), $\delta T_{0,7} T_{0,7}^{-1}$ can be written as

$$
\delta T_{0,7} T_{0,7}^{-1}=\log \left(T_{0,7}^{\mathrm{a}} T_{0,7}^{-1}\right)
$$

where $T_{0,7}^{a}$ denotes the actual position and posture. Components of $\operatorname{SE}(3)$ can be expressed by $\delta \widehat{p}_{i} \mapsto \delta p_{i} \in \Re^{6 \times 1}$ and $\log \left(T_{0,7}^{\mathrm{a}} T_{0,7}^{-1}\right) \mapsto \log \left(T_{0,7}^{\mathrm{a}} T_{0,7}^{-1}\right)^{\vee} \in \mathfrak{R}^{6 \times 1}$. Then, (15) can be 
TABLE 2: The setting error of the robot.

\begin{tabular}{lccc}
\hline Joint & $\delta p_{i}{ }^{\prime}$ & $\delta s_{i}{ }^{\prime}$ & $\delta q_{i}{ }^{\prime}$ \\
\hline $0-1$ & $(0,0.001,0.002,2,1.5,0.5)^{T}$ & $(0,0,0,0, \sin (0.01),-1+\cos (0.01))^{T}$ & 0 \\
$1-2$ & $(0,0.002,0.003,-2,1.2,2.4)^{T}$ & $(0,0,0,0,-\sin (0.015),-1+\cos (0.015))^{T}$ & 0.03 \\
$2-3$ & $(0.001,0.004,0.003,2,-1,2)^{T}$ & $(0,0,0, \sin (0.023), 0,-1+\cos (0.023))^{T}$ & 0.01 \\
$3-4$ & $(0.001,0.005,0.002,2,1.5,-1)^{T}$ & $(0,0,0,-\sin (0.004), 0,-1+\cos (0.004))^{T}$ & 0.02 \\
$4-5$ & $(0.002,0.003,0,-1,1.6,2.3)^{T}$ & $(0,0,0,0, \sin (0.031),-1+\cos (0.031))^{T}$ & 0.015 \\
$5-6$ & $(0.003,0.001,-0.004,2,3,1)^{T}$ & $(0,0,0, \sin (0.013), 0,-1+\cos (0.013))^{T}$ & 0.02 \\
$6-7$ & $(0.005,0.002,-0.003,1.6,2,3.2)^{T}$ & - & - \\
\hline
\end{tabular}

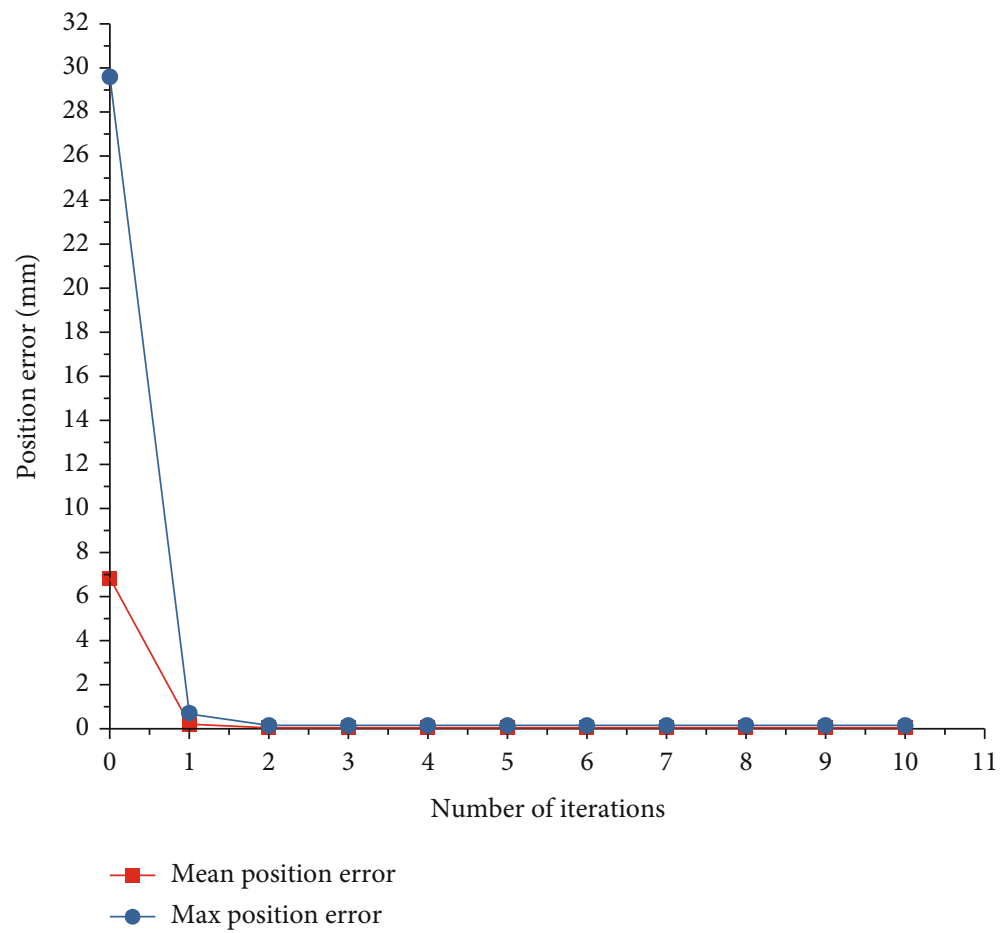

Figure 6: The process of iterative convergence.

rewritten as

$$
\begin{aligned}
\log \left(T_{0,7}^{\mathrm{a}} T_{0,7}^{-1}\right)^{\vee}= & \operatorname{Ad}_{T_{0,1}(0)} \delta p_{1}+\operatorname{Ad}_{T_{0,1}} \operatorname{Ad}_{T_{1,2}(0)} \delta p_{2}+\cdots \\
& +\operatorname{Ad}_{T_{0,6}} \operatorname{Ad}_{T_{6,7}(0)} \delta p_{7}
\end{aligned}
$$

where $T_{0,7}=e^{\widehat{p}_{1}} e^{\hat{s}_{1} q_{1}} e^{\widehat{p}_{2}} e^{\hat{s}_{2} q_{2}} \cdots e^{\hat{p}_{6}} e^{\hat{s}_{6} q_{6}} e^{\widehat{p}_{7}}$ denotes the transformation from coordinate system $0^{\text {th }}$ to $7^{\text {th }}$. The initial position and posture transformation of linkage $i$ relatively to $i-1$ is

$$
T_{i-1, i}^{\mathrm{c}}(0)=e^{\widehat{p}_{i}} e^{\delta \widehat{p}_{i}}=T_{i-1, i}(0) e^{\delta \widehat{p}_{i}}
$$

(18) can be written in the form of linear equations:

$$
y=A x,
$$

where

$$
\begin{gathered}
y=\log \left(T_{0,7}^{\mathrm{a}} T_{0,7}^{-1}\right)^{\vee} \in \mathfrak{R}^{6 \times 1}, \\
x=\left[\delta p_{1}, \delta p_{2}, \cdots, \delta p_{7}\right]^{\mathrm{T}} \in \mathfrak{R}^{6(7) \times 1}, \\
A=\left[\operatorname{Ad}_{T_{0,1}(0)}, \operatorname{Ad}_{T_{0,1}} \operatorname{Ad}_{T_{1,2}(0)}, \cdots, \operatorname{Ad}_{T_{0,6}} \operatorname{Ad}_{T_{6,7}(0)}\right] \in \mathfrak{R}^{6 \times 6 \times 7} .
\end{gathered}
$$

$T_{0,7}^{-1}$ and $A$ can be obtained from the nominal model. $T_{0,7}^{a}$ can be obtained by measuring, usually a laser tracker. $x$ denotes the error of the kinematic parameters to be identified. To improve the accuracy and robustness of the identification, it is usually necessary to measure the position and posture of the tool under different configurations of the robot. If $m$ times of measuring are required, at the $i_{-}{ }^{\text {th }}$ 


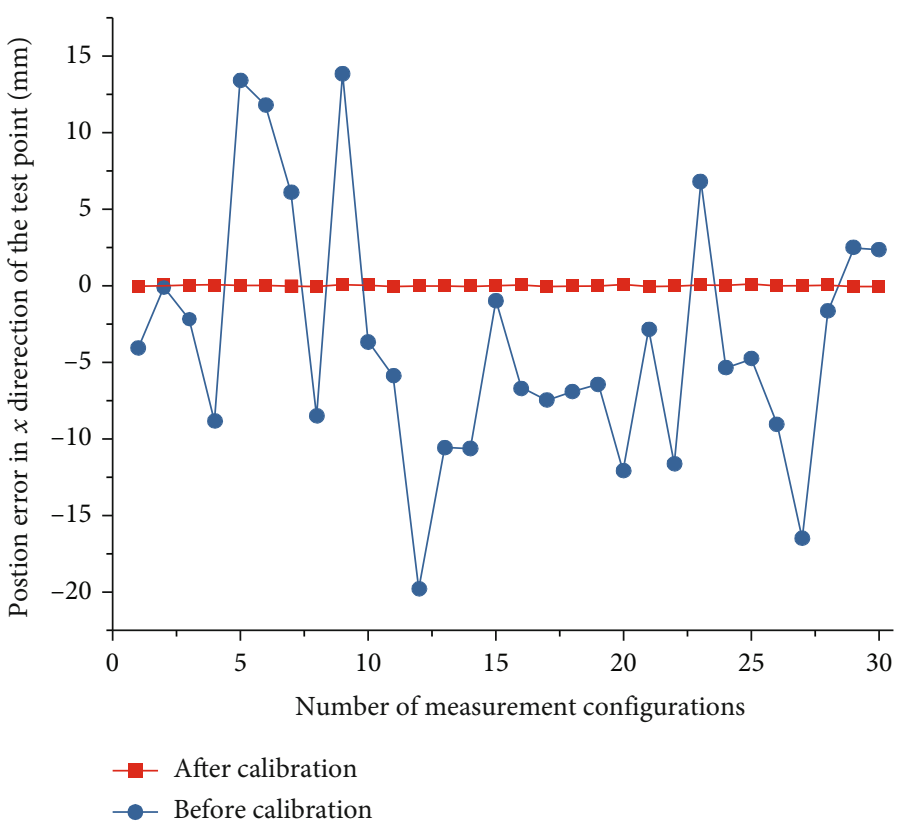

(a) The position error in the $x$ direction

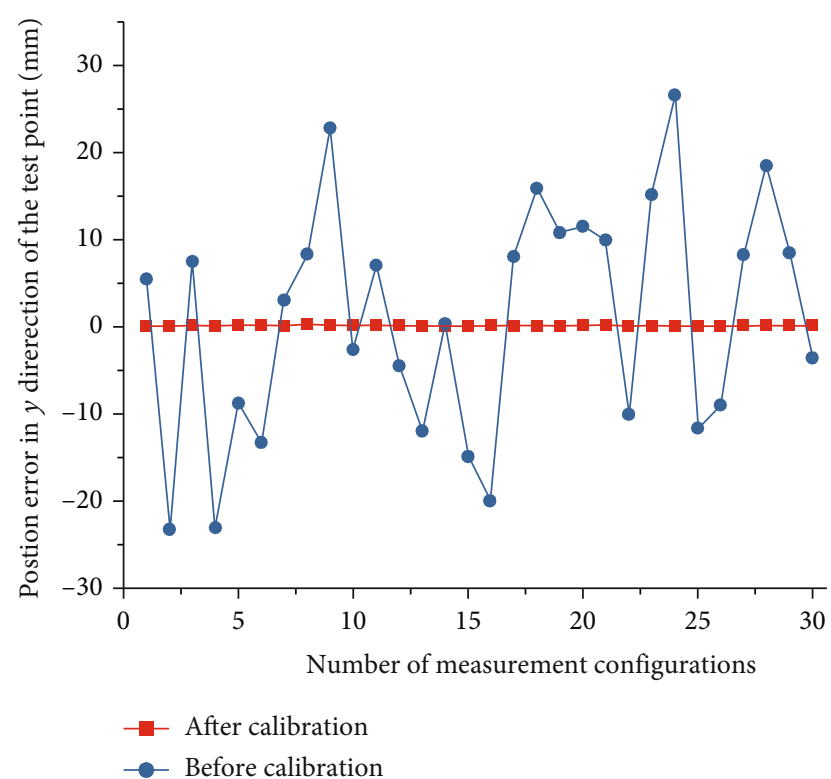

(b) The position error in the $y$ direction

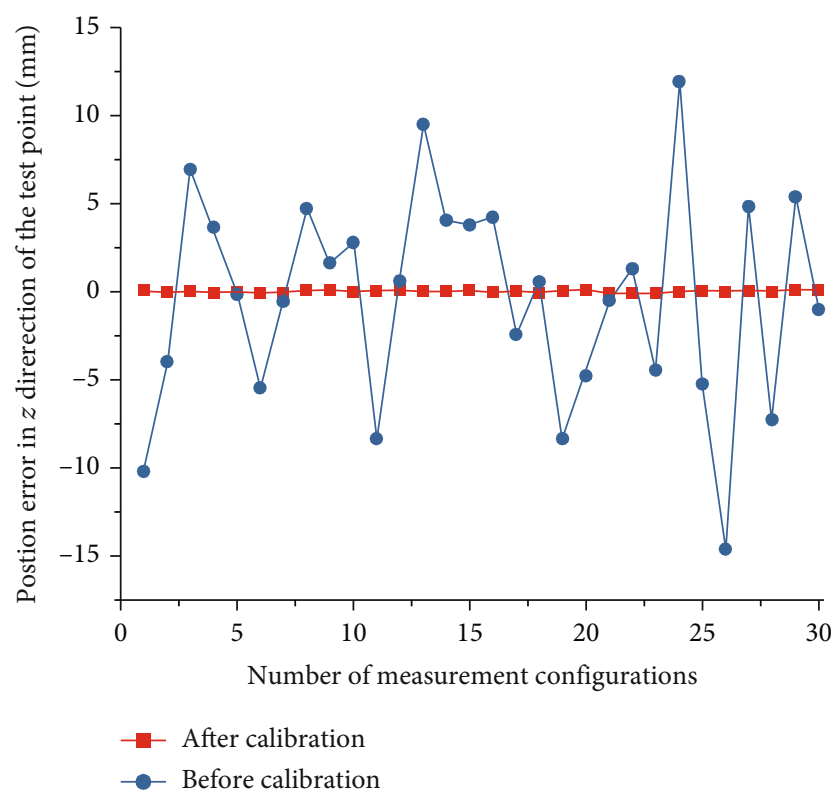

(c) The position error in the $z$ direction

Figure 7: The position error in directions of $x, y$, and $z$ before and after calibration in simulations.

time, the position and posture error $y_{i}$ and the corresponding adjoint mapping matrix $A_{i}$ can be obtained. After finishing acquiring $m$ measuring data, $y_{i}$ and $A_{i}$ are substituted into (22).

$$
Y=\left[\begin{array}{c}
y^{1} \\
\vdots \\
y^{m}
\end{array}\right]=\left[\begin{array}{c}
\mathrm{A}^{1} \\
\vdots \\
A^{m}
\end{array}\right] x=A x,
$$

where

$$
\begin{gathered}
Y=\left[y_{1}, y_{2}, \cdots, y_{m}\right]^{\mathrm{T}} \in \mathfrak{R}^{6 m \times 1}, \\
x=\left[\delta p_{1}, \delta p_{2}, \cdots, \delta p_{7}\right]^{\mathrm{T}} \in \mathfrak{R}^{6 \times 7 \times 1}, \\
A=\left[A_{1}, A_{2}, \cdots, A_{m}\right] \in \mathfrak{R}^{6 m \times 6 \times 7} .
\end{gathered}
$$

Since the model consists of $6 \times m$ linear equations and variables, when $m>7$, the linear least squares algorithm can be used for parameter identification, and the least 
squares solution of $x$ is

$$
x=\left(A^{\mathrm{T}} A\right)^{-1} A^{\mathrm{T}} Y,
$$

where $\left(A^{T} A\right)^{-1} A^{T}$ is the pseudoinverse of $A$.

$T(0)$ can be initialized with the nominal value, i.e., $T(0)$ $=\left[T_{0,1}(0), T_{1,2}(0), \cdots, T_{6,7}(0)\right]^{T}$. $x$ can be calculated by acquired data and (24). Then, $T(0)$ can be updated by (19) till the objective function approaches 0 or $T(0)$ converges to a stable value. Then, the stable $T(0)$ is set as the initial position and posture, i.e., $T(0)=\left[T_{0,1}(0)^{c}, T_{1,2}(0)^{c}, \cdots, T_{6,7}(0)^{c}\right]^{T}$. (7) can be written as (25) after the nominal position and posture matrix is substituted with the above identified one.

$$
T_{0,7}=T_{0,1}^{c}(0) e^{\widehat{s}_{1} q_{1}} T_{1,2}^{c}(0) e^{\hat{s}_{2} q_{2}} \cdots T_{5,6}^{c}(0) e^{\widehat{s}_{6} q_{6}} T_{6,7}^{c} .
$$

3.3. Error Modeling Based on Position. In practice, the posture of the robot is very difficult to obtain, which is time consuming and complicated. One error modeling method based on position is presented in this paper.

Right multiplying $P_{0}$ both sides of (13), one can get

$$
\left[\begin{array}{l}
P \\
1
\end{array}\right]=T_{0,7} P_{0}=e^{p_{1}} e^{\widehat{s}_{1} q_{1}} e^{p_{2}} e^{\widehat{s}_{2} q_{2}} \cdots e^{p_{6}} e^{\widehat{s}_{6}} q_{6} e^{p_{7}} P_{0}
$$

where $P$ denotes the position of the tool, $P_{0}=[0,0,0,1]^{T}$. Then, the position error model can be obtained by differentiating (26):

$$
\left[\begin{array}{c}
\delta P \\
0
\end{array}\right]=\delta T_{0,7}\left[\begin{array}{c}
\mathrm{P}_{0} \\
1
\end{array}\right]+T_{0,7}\left[\begin{array}{c}
\delta P_{0} \\
0
\end{array}\right] .
$$

According to the assumptions above, i.e., the kinematic error only exists in the initial position and posture of the local coordinate system $T(0)$, (27) can be written as

$$
\begin{aligned}
{\left[\begin{array}{c}
\delta P \\
0
\end{array}\right] } & =\delta T_{0,7}\left[\begin{array}{c}
\mathrm{P}_{0} \\
1
\end{array}\right]=\left(\delta T_{0,7} T_{0,7}^{-1}\right) T_{0,7}\left[\begin{array}{c}
\mathrm{P}_{0} \\
1
\end{array}\right] \\
& =\left(\delta T_{0,7} T_{0,7}^{-1}\right)\left[\begin{array}{l}
\mathrm{P} \\
1
\end{array}\right]=\left[\begin{array}{cc}
-\widehat{P} & I_{3} \\
0 & 0
\end{array}\right]\left(\delta T_{0,7} T_{0,7}^{-1}\right)^{\vee} .
\end{aligned}
$$

By substituting (18) into (28), one can get

$$
\delta P=\left[-\widehat{P}_{3}\right]\left(\operatorname{Ad}_{T_{0,1}(0)} \delta p_{1}+\operatorname{Ad}_{T_{0,1}} \operatorname{Ad}_{T_{1,2}(0)} \delta p_{2}+\cdots+\operatorname{Ad}_{T_{0,6}} \operatorname{Ad}_{T_{6,7}(0)} \delta p_{7}\right) .
$$

(29) can be simplified as

$$
y=J x,
$$

where $\mathrm{y}=\delta P=P_{a}-P_{n} \in \mathfrak{R}^{3 \times 1}, P_{n}$ denotes the nominal position, $P_{a}$ denotes the actual position, $x=\left[\delta p_{1}, \delta p_{2}, \cdots \delta p_{7}\right]^{T} \epsilon$

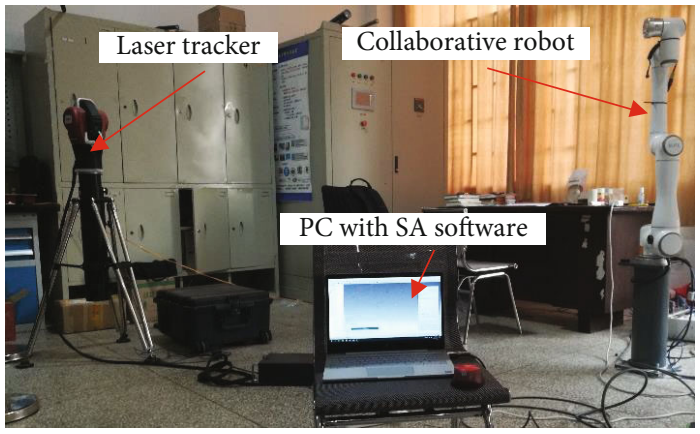

FIgURE 8: The experimental system of calibration for the collaborative robot.

$\mathfrak{R}^{6 \times 7 \times 1}$, and $J=\left[\begin{array}{ll}-\widehat{P} & I_{3}\end{array}\right]\left[A d_{T_{0,1}(0)}, A d_{T_{0,1}} A d_{T_{1,2}(0)}, \cdots A d_{T_{0,6}}\right.$ $\left.A d_{T_{6,7}(0)}\right] \in \mathfrak{R}^{6 \times 6 \times 7}$.

The position of the tool can be measured $m$ times at different configurations. Then, with these measured data, the kinematic parameter identification equations can be obtained as shown in (31).

$$
Y=\left[\begin{array}{c}
y^{1} \\
\vdots \\
y^{m}
\end{array}\right]=\left[\begin{array}{c}
J^{1} \\
\vdots \\
J^{m}
\end{array}\right] x=J x .
$$

One can solve (31) by the iterative least square method with the termination condition of the iteration as $\|x\|<\varepsilon$, as shown in Figure 5. And the least square solution is

$$
x=\left(J^{T} J\right)^{-1} J^{T} Y .
$$

\section{Simulations}

In the simulation, the twists are set as Figure 3, and the kinematic parameters are set as Table 1 . To simulate the measuring error and test the stability of the error model, Gaussian noise with an expectation of 0 and a variance of 0.2 is applied to the data of positions calculated by the kinematic model of the robot. The settings when calculated the data of positions are the following: (1) the errors of kinematic parameters are $\delta \mathrm{p}_{i}{ }^{\prime}, \delta q_{i}{ }^{\prime}$, and $\delta s_{i}{ }^{\prime}$, and (2) the actual initial position and posture is set as follows:

$$
T_{i-1, i}^{\mathrm{a}}(0)=T_{i-1, i}(0) e^{\delta \hat{p}_{i}^{\prime}}(i=1,2, \cdots, 7) .
$$

The actual position is calculated by

$$
P_{0,7}^{\mathrm{a}}=\prod_{i=1}^{6}\left(T_{i-1, i}^{\mathrm{a}}(0) e^{\left(\hat{s}_{i}+\delta \hat{s}_{i}^{\prime}\right)\left(q_{i}+\delta q_{i}{ }^{\prime}\right)}\right) T_{6,7}^{\mathrm{a}}(0) P_{0} .
$$

All the joints of the robot are one freedom; so, the setting error should meet: $\left\|w_{i}+\delta w_{i}^{\prime}\right\|=1,\left(w_{i}+\delta w_{i}^{\prime}\right)^{T}\left(v_{i}+\delta v_{i}^{\prime}\right)$ $=0, s_{i}=\left(v_{i}, w_{i}\right)^{T}$, and $\delta s_{i}^{\prime}=\left(\delta v_{i}^{\prime}, \delta w_{i}^{\prime}\right)^{T}$, as shown in Table 2. 


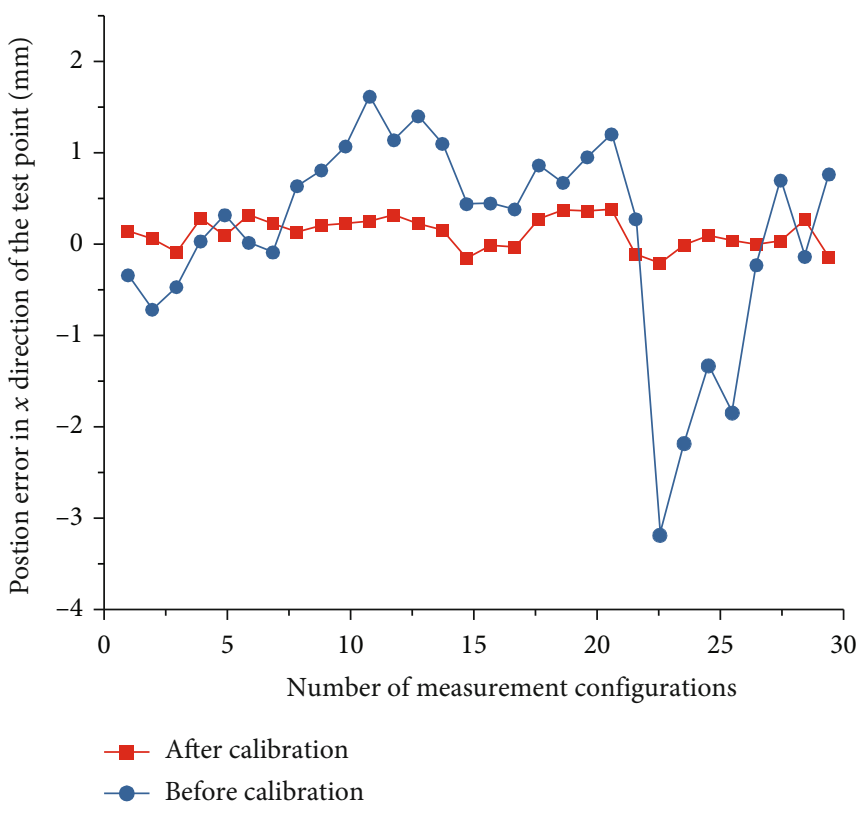

(a) The position error in the $x$ direction

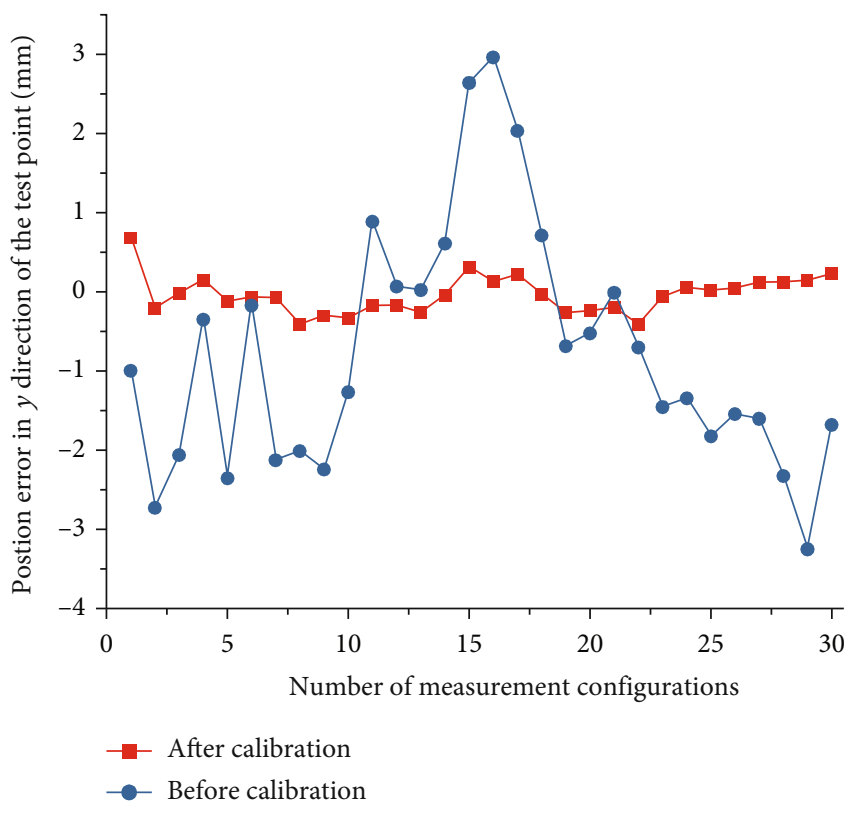

(b) The position error in the $y$ direction

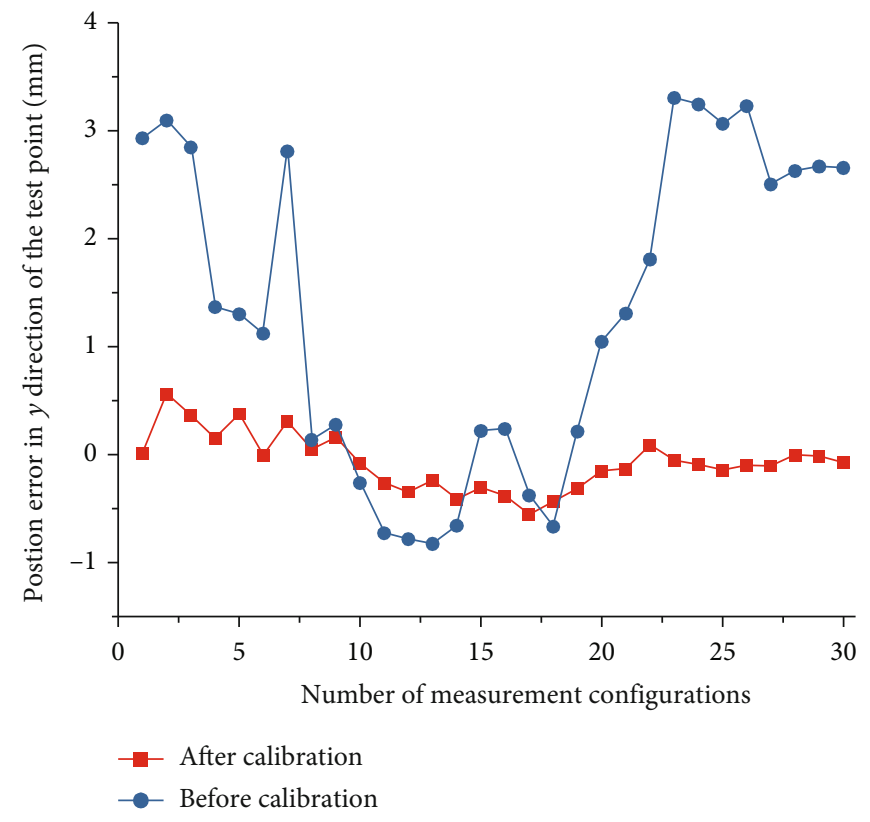

(c) The position error in the $z$ direction

FIGURE 9: The position error in directions of $x, y$, and $z$ before and after calibration in experiments.

100 groups of joint angle data are randomly generated, which are substituted to (34) to obtain the actual position data. The position data are divided into two groups: identification group and verification group. With the identification group of position data, the process of iterative convergence is shown in Figure 6 which shows that after $1^{\text {st }}$ iteration, the objective function is reduced greatly; after 10 iterations, the maximum error is reduced from $29.597 \mathrm{~mm}$ to $0.146 \mathrm{~mm}$, and the average error is reduced from $6.831 \mathrm{~mm}$ to $0.037 \mathrm{~mm}$. The identified parameters are substituted to the kinematic model of the robot to calculate the position error in the three directions of the coordinate system with the verification group of data, and the results are shown in Figure 7 which shows that the maximum error in $x, y$, and $z$ directions is reduced from $19.797 \mathrm{~mm}$ to $0.094 \mathrm{~mm}$, $26.548 \mathrm{~mm}$ to $0.092 \mathrm{~mm}$, and $14.626 \mathrm{~mm}$ to $0.095 \mathrm{~mm}$, respectively.

\section{Experiments}

To further verify the calibration method based on position information and LPoE model proposed in this paper, a calibration experiment was carried out on a six degree-offreedom collaborative robot, the repeated positioning 
accuracy of which is $0.03 \mathrm{~mm}$. The measuring instrument is RADIAN Pro R20 of API, and the measuring radium and accuracy are $20 \mathrm{~m}$ and $15 \mu \mathrm{m}+0.7 \mu \mathrm{m} / \mathrm{m}$, respectively. The measuring software is Spatial Analyzer (SA) of New River Kinematics. The experimental system is shown in Figure 8.

100 groups of joint angles are generated in the measurable space of the laser tracker, 70 groups of which are identification sets and the other 30 groups are verification sets. According to Figure 5, the kinematic parameters are identified, and the verification results are shown in Figure 9. The results show that after calibration, the maximum error in $x$ , $y$, and $z$ directions is reduced from $3.171 \mathrm{~mm}$ to $0.393 \mathrm{~mm}, 3.258 \mathrm{~mm}$ to $0.678 \mathrm{~mm}$, and $3.297 \mathrm{~mm}$ to $0.560 \mathrm{~mm}$, respectively. Therefore, the accuracy of the collaborative robot is increased greatly by the proposed calibration method based on position data and improved LPoE.

\section{Conclusions}

The kinematic model of the collaborative robot based on the LPoE model was established, and the corresponding error model and calibration method were derived. The calibration method was verified by simulation, which proved the validity of the error model and the calibration method. A six degree-of-freedom collaborative robot was used as the experimental objective, and the API RADIAN Pro R20 laser tracker was used as the measurement equipment to perform kinematic parameter calibration experiments which show that the positioning errors of the six degree-of-freedom collaborative robot decrease from $3.171 \mathrm{~mm}, 3.258 \mathrm{~mm}$, and $3.297 \mathrm{~mm}$ to $0.393 \mathrm{~mm}, 0.678 \mathrm{~mm}$, and $0.560 \mathrm{~mm}$ in the directions of $x, y$, and $z$, respectively, after kinematic parameter calibration. Therefore, the proposed calibration method is effective.

However, the residuals are still about $0.6 \mathrm{~mm}$ after calibration. The main reason is that kinematic calibration cannot eliminate the influence of nongeometric errors such as the elastic deformation of the joint. In the future, relevant research work will be carried out on nongeometric errors to further improve the positioning accuracy of the robot.

\section{Data Availability}

Data were curated by the authors and are available upon request.

\section{Conflicts of Interest}

The authors declare that there is no conflict of interest regarding the publication of this paper.

\section{Acknowledgments}

This work was supported by the National Natural Science Foundation of China (Grant No. 51865020).

\section{References}

[1] ISO, TS 15066: 2016: Robots and robotic devices-Collaborative robots, International Organization for Standardization, Geneva, Switzerland, 2016.

[2] L. Fu, R. Wu, and J. Zhao, "The evolution and enlightenment of safety specification of cooperative robots: ISO/TS 15066," Robot, vol. 39, no. 4, pp. 532-540, 2017.

[3] Q. Liu, A. Liu, W. Meng, Q. Ai, and S. Q. Xie, "Hierarchical compliance control of a soft ankle rehabilitation robot actuated by pneumatic muscles," Frontiers in Neurorobotics, vol. 11, p. 64, 2017.

[4] W. Liu, D. Chen, and L. Zhang, "Trajectory generation and adjustment method for robot manipulators in human-robot collaboration," Robot, vol. 38, no. 4, pp. 504-512, 2016.

[5] X. Liu, F. Xie, and J. Wang, "Current opportunities in the field of mechanisms in China," Journal of Mechanical Engineering, vol. 51, no. 13 , pp. 2-12, 2015.

[6] L. Li, Z. Luo, F. X. He, K. Sun, and X. L. Yan, "An improved partial similitude method for dynamic characteristic of rotor systems based on Levenberg-Marquardt method," Mechanical Systems and Signal Processing, vol. 165, article 108405, 2022.

[7] X. Liu, Q. Liu, C. Yue et al., "Intelligent machining technology in cutting process," Journal of Mechanical Engineering, vol. 54, no. 16, pp. 45-61, 2018.

[8] Z. Fu, J. S. Dai, K. Yang, X. Chen, and P. López-Custodio, "Analysis of unified error model and simulated parameters calibration for robotic machining based on Lie theory," Robotics and Computer-Integrated Manufacturing, vol. 61, article 101855, 2020.

[9] W. Gao, H. Wang, Y. Jiang, and X. Pan, "Research on the calibration for a modular robot," Journal of Mechanical Engineering, vol. 50, no. 3, pp. 33-40, 2014.

[10] B. Tipary and G. Erdős, "Generic development methodology for flexible robotic pick-and-place workcells based on Digital Twin," Robotics and Computer-Integrated Manufacturing, vol. 71, article 102140, 2021.

[11] Z. Xie, S. Xin, X. Li, and S. Xu, "Method of robot calibration based on monocular vision," Journal of Mechanical Engineering, vol. 47, no. 5, pp. 35-39, 2011.

[12] Z. Jiang, W. Zhou, H. Li, Y. Mo, W. Ni, and Q. Huang, “A new kind of accurate calibration method for robotic kinematic parameters based on the extended Kalman and particle filter algorithm," IEEE Transactions on Industrial Electronics, vol. 65 , no. 4 , pp. 3337-3345, 2017.

[13] W. Li, "Analysis on Kinematic Model of Welding Robot Calibration for Automobile Fittings," in 8th International Conference on Applied Science, Engineering and Technology (ICASET), pp. 146-150, Qingdao, China, 2018.

[14] Y. Lu, L. Yu, and B. Guo, "Calibration of industrial robot structure parameters based on closed dimensional chain," Chinese Journal of Scientific Instrument, vol. 39, no. 2, pp. 38-46, 2018.

[15] K. Okamura and F. Park, "Kinematic calibration using the product of exponentials formula," Robotica, vol. 14, no. 4, pp. 415-421, 1996.

[16] R. He, Y. Zhao, F. Han, S. Yang, and S. Yang, "Experimentation on identifying the kinematic parameters of serial mechanism based on the product-of-exponential formula," Robot, vol. 33, no. 1, p. 35, 2011.

[17] R. He, Y. Zhao, S. Yang, and S. Yang, "Kinematic-parameter identification for serial-robot calibration based on POE 
formula," IEEE Transactions on Robotics, vol. 26, no. 3, pp. 411-423, 2010.

[18] Y. Jiang, L. Yu, Y. Chang, H. Jia, and H. Zhao, "Robot calibration based on modified differential evolution algorithm," Optics and Precision Engineering, vol. 29, no. 7, pp. 15801588, 2021.

[19] Y. Wang, Q. Wei, C. Hu, and X. Ding, "A self-calibration method of space manipulators based on POE formula," Journal of Beijing University of Aeronautics and Astronautics, vol. 44, no. 11, pp. 2336-2342, 2018.

[20] G. Chen, Q. Jia, T. Li, and H. Sun, "Calibration method and experiments of robot kinematics parameters based on error model," Robot, vol. 34, no. 6, pp. 680-688, 2012.

[21] I. Chen, G. Yang, C. T. Tan, and S. H. Yeo, "Local POE model for robot kinematic calibration," Mechanism and Machine Theory, vol. 36, no. 11-12, pp. 1215-1239, 2001.

[22] R. M. Murray, Z. Li, and S. S. Sastry, A mathematical introduction to robotic manipulation, CRC, Boca Raton, FL, USA, 1994. 